\title{
POTENCIALIDADES GEOGRÁFICAS DE LA LAGUNA DE LAS PEONÍAS (VENEZUELA) COMO MUSEO NATURAL PARA LA ENSEÑANZA DE LA GEOGRAFÍA FÍSICA
}

Ramón Labarca ${ }^{1}$; Belmary Barreto ${ }^{1}$; Jorge Bernal ${ }^{1}$

Recibido: 25/02/2018

Aceptado: 01/09/2018

\section{RESUMEN:}

Se propone utilizar las potencialidades geográficas del escenario de la Laguna de las Peonías como museo natural para la enseñanza de la Geografía Física. La metodología es descriptiva, proyectiva, bajo un diseño de campo, no experimental. Al diagnosticar los "Recursos didácticos", más del $80 \%$ de los encuestados afirma que el docente no utiliza "recursos dirigidos y naturales" en la enseñanza. Respecto al "nivel de conocimiento", existe debilidad en el dominio del mismo, más del 75\% de los estudiantes no acertaron en los indicadores "presencia de albuferas", "presencia de playas y dunas costeras" y "procesos de sedimentación" de la Laguna de las Peonías. Se diseñó una propuesta de museo natural basado en el escenario de la referida laguna, materializado en una visita guiada que contempla cinco (5) estaciones.

\section{Palabras Clave:}

Laguna de las Peonías, museo natural, Geografía Física, visita guiada.

${ }^{1}$ Centro de Estudios Geográficos, Facultad de Humanidades y Educación, Universidad del Zulia, Venezuela. 


\section{Abstract:}

It is proposed to use the geographical potentialities of the Peonies lagoon scenario as a natural museum for the teaching of Physical Geography. The methodology is descriptive, projective, under a field design, not experimental. When diagnosing the «didactic resources», more than $80 \%$ of the respondents affirmed that the teacher does not use «directed and natural resources» in teaching. Regarding the «level of knowledge», there is weakness in the domain of the same, more than $75 \%$ of students did not succeed in the indicators «presence of lagoons», "presence of beaches and coastal dunes» and «sedimentation processes» of the Peonies lagoon. A proposal for a natural museum was designed based on the scenario of the referred lagoon, materialized in a guided tour that includes five (5) stations.

\section{KeYWORDS:}

Laguna de las Peonías, natural museum, physical geography, guided tour.

\section{RÉSUMÉ:}

Il est proposé d'utiliser les potentialités géographiques du scénario Laguna de las Peonías comme un musée naturel pour l'enseignement de la géographie physique. La méthodologie est descriptive, projective, sous une conception de terrain, pas expérimentale. Lors du diagnostic des «ressources didactiques», plus de $80 \%$ des personnes interrogées ont affirmé que l'enseignant n'utilise pas de «ressources dirigées et naturelles» dans l'enseignement. En ce qui concerne le "niveau de connaissance", il y a faiblesse dans le domaine de la même chose, plus de $75 \%$ des étudiants n'ont pas réussi dans les indicateurs "présence de lagunes", "présence de plages et dunes côtières" et "processus de sédimentation" Laguna de las Peonías. Une proposition pour un musée naturel a été conçue sur la base du scénario du lagon référé, matérialisé dans une visite guidée qui comprend cinq (5) stations.

\section{Mots-CLÉs:}

Laguna de las Peonías, musée naturel, géographie physique, visite guidée.

\section{INTRODUCCIÓN}

En pleno siglo XXI, se sigue notando en los planteles educativos públicos y privados cómo los docentes de Geografía se apegan al libro de texto como único recurso instruccional y pauta para el desarrollo de las sesiones escolares (Gouveia, Montiel y Bejas 2005), dejando a un lado la innumerable gama de recursos y estrategias que tienen a sus disposición. Ante tal situación, emerge la necesidad de 
replantear el método de clases utilizado por los profesionales de la docencia en el área de la Geografía, buscando con ello enfocarse en la práctica escolar cotidiana de los estudiantes.

Sin embargo, la actual disposición de los docentes en la enseñanza de la ciencia geográfica dista mucho de ser lo que realmente implica los alcances científicos de ella. Santiago (2005), es enfático al alegar que a pesar de los avances tecnológicos y científicos, la práctica pedagógica de la Geografía hoy por hoy se sigue abordando desde una concepción descriptiva de los fenómenos naturales y sociales, alejada de la identificación de hechos geográficos desde la inmediatez espacial de los educandos.

Ante esta situación, pensadores contemporáneos proponen la utilidad del paisaje natural cercano al estudiante para el aprendizaje de los contenidos relacionados a la Geografía, entre ellos destacan: Montiel y González (2012); Labarca y Chourio (2016); Barreto y Bernal (2016); entre otros. Las expectativas puestas en la utilización del paisaje para aplicarlo a los objetivos de enseñanza, conlleva a generar en los educandos la protección por la naturaleza y el cuido por el patrimonio natural de su entorno (Zoido, 2010).

El paisaje natural, como escenario de enseñanza, juega un papel determinante en la transmisión de los contenidos relacionados a la Geografía, sobre todo los que se vinculan con la Geografía Física, ya que coloca de manifiesto y a la vista de los estudiantes realidades geográficas que son abstractas como relieve, vegetación, clima, tiempo atmosférico, estructuras geológicas, procesos exógenos de la tierra, entre otros. Por tal razón, el adecuado uso del paisaje natural como escenario didáctico prevé a los docentes un verdadero museo natural de enseñanza.

La utilidad del museo natural en la enseñanza de la Geografía resulta atractiva, dinámica y pertinente respecto a su incorporación en la praxis escolar docente, puesto que brinda una herramienta sustancial para el aprendizaje de los contenidos referentes a la Geografía Física. A lo tocante, Zoido (2010), increpa que utilizar el paisaje natural incentiva en los estudiantes el cuidado y preservación del patrimonio natural de su entorno, y ello se logra a partir de la compresión de los elementos físicos que lo conforman.

Así pues, el paisaje natural se asemeja a un museo en el cual se pueden observar ordenadamente la piezas (elementos físicos y sociales) formando un solo conjunto, y sirve de museo didáctico para la enseñanza de las interrelaciones geográficas del medio de una manera directa, clara, empírica y tangible. En concordancia, Labarca y Chourio (2016) y Barreto y Bernal (2016), hacen mención al paisaje como recurso de enseñanza abre la posibilidad de que el docente muestre los contenidos de Geografía directamente en la realidad donde ocurren, alcanzando así aprendizajes significativos en sus estudiantes. 
Para Negrete y Montiel (2016), es necesario que las prácticas pedagógicas de la Geografía se reorienten hacia el uso de los paisajes in situ con el objeto de fomentar en los educandos una conciencia crítica de su realidad espacial y desarrollar en él sentimientos de valoración por los espacios naturales. Además de ello, se logra vincular al estudiante con la realidad nacional, regional y local, por lo que estudiar Geografía es propicio a la hora de formar ciudadanos de calidad desde el plano educativo.

Los estudiantes al verse inmiscuidos en su entorno, lo descubren y exploran por sí mismos, propiciando en ellos un aprendizaje activo, ya que no sólo se interesa por observar los elementos naturales sino que se ha de preocupar por darle explicación y relación; e integrador, puesto que lograrán obtener una visión global del espacio geográfico que les rodea (García, Jiménez y Rodríguez, 2009). Así pues, la utilidad del paisaje como escenario didáctico debe ser parte de los elementos a utilizar por el docente en la enseñanza de la Geografía en Educación Media General, planificando para ello una adecuada estrategia y un novedoso recurso didáctico.

Montiel y González (2012), hacen mención a la importancia que tiene la elección de metodología adecuadas para la visita y estudio de los paisajes, ya que éstos son espacios donde los estudiantes pueden alcanzar competencias cognitivas para observar, describir, comparar, analizar y comprender la génesis de los elementos naturales y la incidencia de la sociedad en ellos. Bajo esta premisa, los itinerarios geográficos, visitas guiadas, excursiones y museos naturales, serian opciones ideales para implementar el trabajo de campo hacia paisajes locales.

En Venezuela, la región zuliana cuenta con numerosos paisajes que poseen potencialidades geográficas dignas de estudiar, explorar y comprender, por lo que deben ser aprovechados como medios educativos mediante la implementación de renovadas estrategias y recursos. De entre los paisajes zulianos destaca el escenario natural de la Laguna de las Peonías, un pintoresco complejo lagunar ubicado al noreste del estrecho de Maracaibo, entre los municipios Maracaibo y Mara, en la parroquia Idelfonso Vázquez (figura 1), situado astronómicamente entre las coordenadas $10^{\circ} 43^{\prime} 10^{\prime \prime}$ y $10^{\circ} 45^{\prime} 50^{\prime}$ " de Latitud Norte y $71^{\circ} 35^{\prime} 02^{\prime}$ ' y $71^{\circ} 40^{\prime} 50^{\prime \prime}$ de Longitud Oeste, contando con $5900 \mathrm{~m}$ de longitud, $2200 \mathrm{~m}$ de anchura y profundidad promedio de $0,70 \mathrm{~cm}$.

La Laguna de las Peonías, está contenida por un abanico de elementos geográficos (albuferas, manglares, clima tropical, procesos de sedimentación, playas, dunas costeras, intervención antrópica, problemáticas socio-ambientales, entre otros) que pueden ser develados a los educandos con la implantación de una apropiada estrategia, renovando así la praxis educativa de la Geografía desde lo local.

Según las consideraciones anteriores, el paisaje de la Laguna de las Peonías adquiere especial significado como escenario didáctico para la enseñanza del medio 
físico local; la estructura geomorfológica de su fisionomía, los procesos exógenos que la modelan y los elementos geográficos que la conforman, hacen de éste espacio un laboratorio de encuentro geográfico donde los educandos adquieren conocimientos empíricos, críticos y analíticos de su entorno inmediato. En función de ello, mediante este artículo, se propone la utilización de las potencialidades geográficas del escenario de la Laguna de las Peonías como un museo natural que dinamiza la enseñanza de la Geografía Física local. La propuesta está materializada por una visita guiada con cinco (5) estaciones sobre el paisaje seleccionado, dirigida a estudiantes del 3er año de Educación Media General.

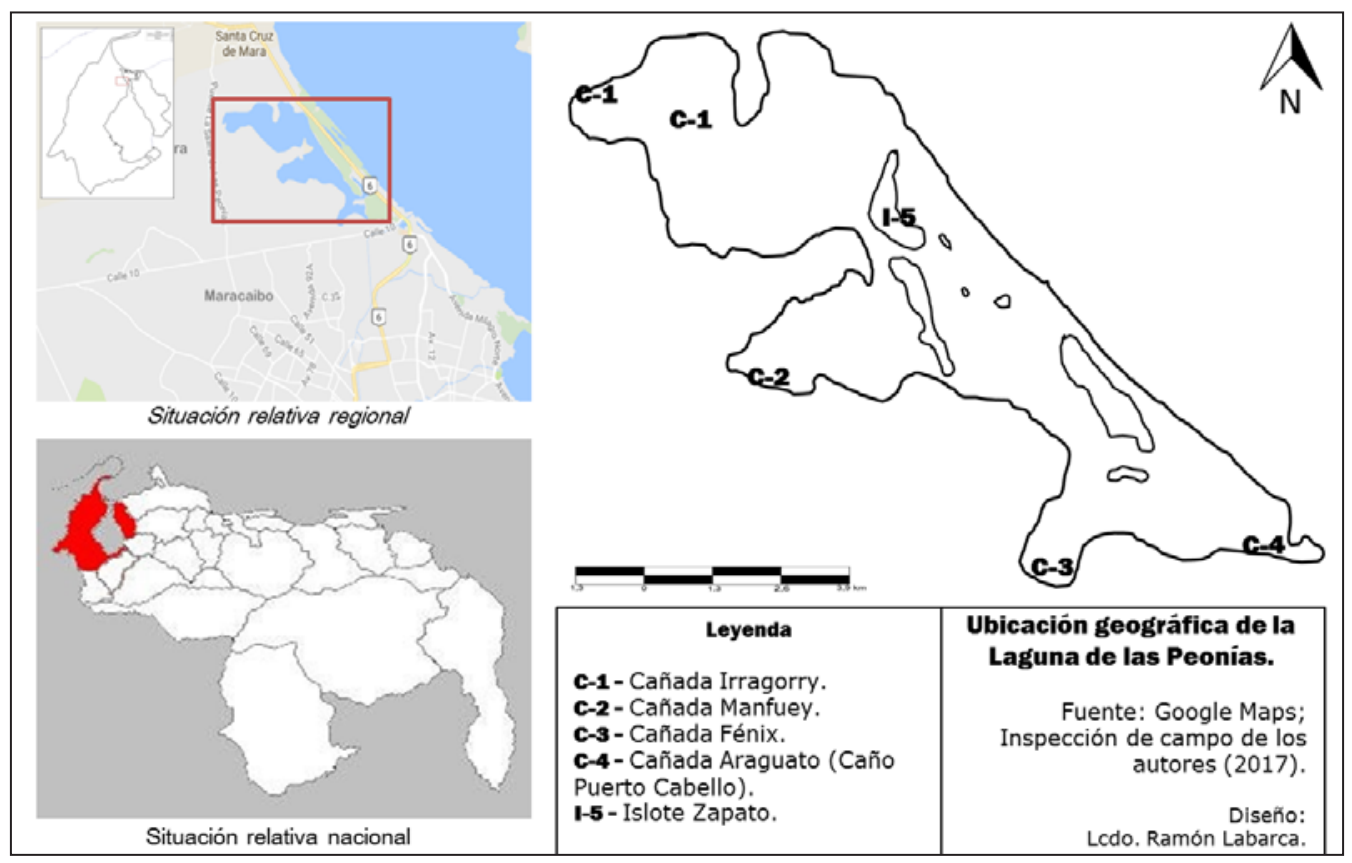

FIgURA 1. Ubicación geográfica de la Laguna de las Peonías.

Fuente: Imágenes de satélite de www.googlemaps.com; Inspección de campo de los autores (2017). Elaboración propia.

\section{METODOLOGÍA}

\subsection{Tipo y diseño de la investigación}

El presente estudio se perfila en las líneas de ser una investigación descriptiva, ya que persigue hacer una descripción en detalle de las potencialidades paisajísticas y geográficas de la Laguna de las Peonías como museo natural para la enseñanza del medio físico en 
la asignatura Geografía de Venezuela y de la realidad educativa que se lleva a cabo en tal asignatura en las instituciones objeto de estudio. En opinión de Hernández, Fernández y Baptista (2014), los estudios de alcance descriptivo buscan especificar las propiedades, características y perfiles del objeto de estudio, representado éste por personas, grupos, comunidades, objetos, fenómenos o paisajes, es decir, únicamente pretenden recoger y medir información de manera independiente.

Así mismo, la propuesta que se deriva de este estudio, catalogada como una visita guiada sobre el escenario natural de la Laguna de las Peonías, lo califica como investigación proyectiva. Para Hurtado (2010), los estudios proyectivos consisten en la construcción de una propuesta, un plan, un programa o un aparato, para dar solución a la problemática de un grupo social o institución en particular en algún área del conocimiento desde el punto de vista práctico. La propuesta, producto de esta investigación, proyecta dar solvencia a la ausencia de estrategias y recursos didácticos basados en paisajes naturales para la enseñanza de la Geografía en las instituciones Liceo Privado "San José de Calasanz" y Unidad Educativa Colegio Adventista "Sierra Maestra", ambas ubicadas en el sector Sierra Maestra del municipio San Francisco, estado Zulia, Venezuela.

La recolección de información en el lugar donde ocurre la problemática de ésta investigación, referida a la ausencia de estrategias y recursos basados en paisajes

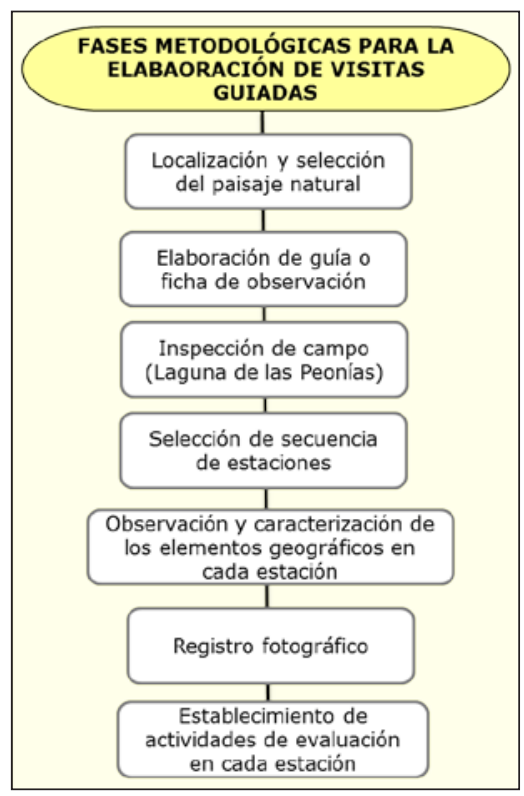

FIgURA 2. Fases para la elaboración de visita guiada al escenario natural de la Laguna de las Peonías. Fuente: Olave (2005); Crespo (2012); Labarca y Chourio (2016). Adaptado por los autores. 
naturales, tipifican al diseño como de campo; y la no manipulación de los datos recabados en el campo, cataloga el estudio como no experimental. La investigación de campo guarda relación con la fase documental en este estudio, mediante la cual se desarrolla el procedimiento metodológico para la elaboración de una visita guiada basada en las potencialidades geográficas de la Laguna de las Peonías como museo natural. La estructura organizativa y metodológica sobre itinerarios geográficos propuesta por Olave (2005), Crespo (2012) y Labarca y Chourio (2016), son la base para la elaboración de la visita guiada al complejo lagunar de las Peonías (figura 2).

\subsection{Población}

La población, considerada censal, está conformada por 124 estudiantes cursantes de la asignatura Geografía de Venezuela para el período escolar 2016-2017, 58 pertenecientes al Liceo Privado "San José de Calasanz" y 66 estudiantes de la Unidad Educativa Colegio Adventista "Sierra Maestra" (tabla 1), ambas entidades ubicadas en el sector Sierra Maestra, parroquia Francisco Ochoa, municipio San Francisco del estado Zulia, Venezuela. Para Chávez (2007), la población censal se refiere a cuando la población de una investigación es reducida y finita, por lo que es determinable, accesible y medible, y puede ser tomada en su totalidad para la recolección de los datos.

\begin{tabular}{|c|c|c|c|}
\hline UNIDAD DE ESTUDIO & ASIGNATURA & GRADO/SECCIÓN/TURNO & ESTUDIANTES \\
\hline \multirow{2}{*}{$\begin{array}{l}\text { Liceo Privado «San } \\
\text { José de Calasanz» }\end{array}$} & \multirow{4}{*}{$\begin{array}{c}\text { Geografía de } \\
\text { Venezuela }\end{array}$} & 3ero. «A»/ Diurno & 28 \\
\hline & & 3ero. «B»/ Diurno & 30 \\
\hline \multirow{2}{*}{$\begin{array}{l}\text { Unidad Educativa } \\
\text { Colegio Adventista } \\
\text { «Sierra Maestra» }\end{array}$} & & 3ro. $\varangle \mathrm{C} » /$ Vespertino & 32 \\
\hline & & 3ro. «D» / Vespertino & 34 \\
\hline & & TOTAL & 124 \\
\hline
\end{tabular}

TABla 1. Población censal de estudio.

Fuente: Listas oficiales de la asignatura Geografía de Venezuela, período escolar 2016-2017.

Elaboración propia.

\subsection{Técnicas e instrumentos de recolección de datos}

La encuesta es la principal técnica utilizada en la fase de recolección de datos en la presente investigación. Sabino (2007), asevera que la encuesta consiste en solicitar información a un grupo socialmente significativo sobre el tema o problemático en 
estudio, lo cual puede ser de manera escrita o verbal. El instrumento seleccionado para dicha técnica, es el cuestionario. Éste, consiste en un conjunto de preguntas referentes a una o más variables a medir, apuntando a ser congruentes con la problemática planteada (Hernández, Fernández y Baptista, 2014).

El cuestionario, de tipo auto-administrado, es aplicado a los estudiantes de las instituciones educativas mencionadas en la tabla 1, en aras de medir las dimensiones "recursos didácticos" utilizados por los docentes en la asignatura Geografía de Venezuela y "nivel de conocimiento" que poseen los estudiantes sobre los elementos geográficos presentes en el escenario de la Laguna de las Peonías como museo natural, contando con un total de 25 ítems con alternativas de respuesta SI-NO (tabla 2).

\begin{tabular}{|l|c|c|}
\hline \multicolumn{1}{|c|}{ Indicador: Presencia de albuferas } & SI & NO \\
\hline $\begin{array}{l}\text { 18.- ¿Una albufera es una laguna costera aislada del mar por un cordón } \\
\text { litoral? }\end{array}$ & & \\
\hline $\begin{array}{l}\text { 19.- ¿Es la Laguna de las Peonías una albufera separada del Lago de } \\
\text { Maracaibo? }\end{array}$ & & \\
\hline Indicador: Presencia de dunas costeras & SI & NO \\
\hline 20.- ¿Las dunas costeras son producto de la acción erosiva de los glaciares? & & \\
\hline 21.- ¿Existen dunas costeras en el paisaje de la Laguna de las Peonías?. & & \\
\hline
\end{tabular}

TABLA 2. Indicadores e ítems que son parte del cuestionario.

Elaboración propia.

Por otro lado, la observación directa es parte de las técnicas empleadas en la inspección de campo. Para ello, el instrumento utilizado es la ficha de observación, siendo una herramienta metodológica que se emplea para hacer anotaciones de campo donde se registran y describen aspectos relacionados a las percepciones, vivencias, expresiones y reflexiones de lo observado (Incart, Fuentelzas y Pulpón, 2006). Con la ficha de observación se registra la presencia de los elementos geográficos, geomorfológicos y ambientales que se exhiben en la Laguna de las Peonías, describiendo sus características primordiales y utilidad didáctica. Aunado a ello, el registro fotográfico es de vital importancia en la recolección de datos visuales sobre los remanentes geográficos presentes en la Laguna de las Peonías y en la selección de las estaciones de la visita guiada como propuesta de esta investigación.

Así mismo, la técnica documental es utilizada para la recolección de fuentes primarias sobre la variable de estudio. El instrumento para la referida técnica es el matriz de registro, en la cual se compilan la serie de libros, artículos, tesis, blogger, entre otros, 
que son el basamento teórico de la investigación. Los instrumentos fueron sometidos a la validez de cuatro (4) expertos de las áreas de Geografía física y didáctica de la Geografía, arrojando como resultado la pertinencia para su aplicación. Además, según fórmula de Kuder Richardson, se obtuvo un valor de $0,83 \%$, lo cual refleja un muy alto grado de confiabilidad (Pérez, 1981; Hernández y col., 2014).

Para la valoración descriptiva e interpretativa de los datos recabados en la dimensión "nivel de conocimiento" que poseen los estudiantes sobre las potencialidades geográficas de la Laguna de las Peonías como museo natural, se utiliza un baremo de interpretación con el cual se confrontan analíticamente los resultados de las respuestas obtenidas, y así manifestar los datos recabados en cada variable e indicador en estudio. El baremo de interpretación, propuesto por Briones (1990), posee carácter arbitrario en la categorización de su escala, tanto en lo que concierne al número de categorías como a los límites numéricos de cada uno de ellas (tabla 3).

\begin{tabular}{|c|c|}
\hline $\begin{array}{c}\text { Límites numéricos } \\
\text { (Intervalos) }\end{array}$ & Categorización \\
\hline Mas o igual al $50 \%$ & Dominio de conocimiento \\
\hline Menos o igual al $49 \%$ & $\begin{array}{c}\text { Debilidad en el dominio del } \\
\text { conocimiento }\end{array}$ \\
\hline
\end{tabular}

TABLA 3. Baremo de interpretación.

Fuente: Briones (1990). Adaptado por los autores.

\section{RESULTADOS Y DISCUSIÓN}

\subsection{La diagnosis}

Para la dimensión "Recursos didácticos" que el docente utiliza para la sesión escolar en la asignatura Geografía de Venezuela (figura 2), se evidencia la ausencia de mapas, carteles, computadoras y radios, ya que más del 55\% de los encuestados apuntan de manera negativa en los indicadores "recursos preparados" y "recursos audiovisuales". Destaca además, que el $68 \%$ de los estudiantes dictamina que el docente hace uso de los "recursos existentes" tales como pizarras, bibliotecas y laboratorios, lo que denota la ausencia de recursos novedosos en la enseñanza y el tradicionalismo en la comunicación del saber.

Por otro lado, más del $80 \%$ de los encuestados afirman que el docente no utiliza los "recursos naturales" y "recursos dirigidos" en la enseñanza (figura 3), es decir, hay nulidad en la aplicación de trabajos de campos, itinerarios geográficos, visitas guiadas o excursiones a paisajes naturales locales, regionales o nacionales como estímulo para el aprendizaje de los contenidos geográficos, lo cual es el eje problema de esta investigación. 
Labarca y Chourio (2016), Barreto y Bernal (2016) y Negrete y Montiel (2016), son incisivos al inferir que la utilidad del paisaje en la enseñanza, implica que el educando verifique contenidos, se vea inmiscuido en su propia realidad geográfica y focalice verdaderos problemas socio-ambientales por los que atraviesa su localidad cercana. En opinión de los investigadores, las visitas guiadas son un novedoso recurso para la identificación de fenómenos geográficos in situ hacia escenarios o paisajes que, en definitiva, fungen como excelentes museos naturales de enseñanza.

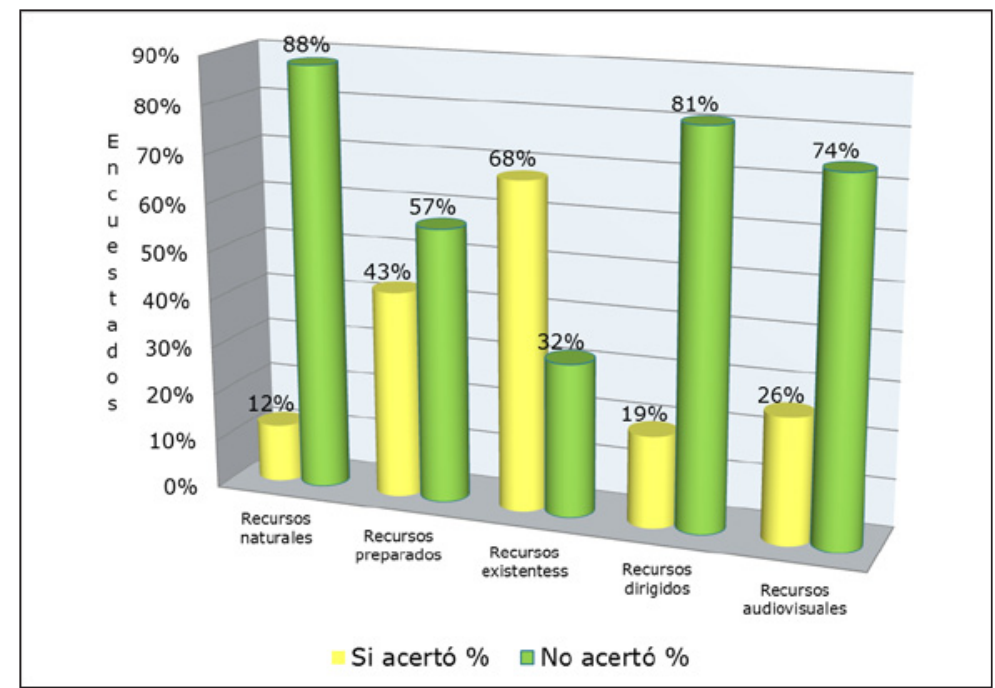

Figura 3. Dimensión: Recursos didácticos.

Elaboración propia.

La dimensión "Nivel de conocimiento", referida a los aprendizajes asertivos y no asertivos que poseen los educandos sobre las potencialidades geográficas presentes en el escenario de la Laguna de las Peonías, se mide contrastando los datos obtenidos con un baremo de interpretación (tabla 3). A lo tocante, resulta evidente la debilidad en el dominio de conocimientos que poseen los dicentes respecto a los "aspectos geográficos" del citado complejo lagunar, puesto que más del $60 \%$ respondió de manera incorrecta a los ítems que agrupan los indicadores "ubicación", "vegetación bosque de manglar", "fauna característica", "profundidad" e "hidrografía" (figura 4), lo cual manifiesta un grave desconocimiento de la Geografía Física de la región zuliana, en específico de paisajes locales como el geosistema lacustre de Las Peonías.

En relación a los ítems que aglomeran los indicadores "presencia de albuferas", "presencia de dunas costeras", "sedimentación" y "degradación antrópica", referente a 
al subdimensión "aspectos geomorfológicos de las Laguna de las Peonías", la tendencia reflejada en la figura 5 , evidencia que más del $75 \%$ de la población de estudio respondió de manera incorrecta. Ello se traduce en debilidad del dominio del conocimiento

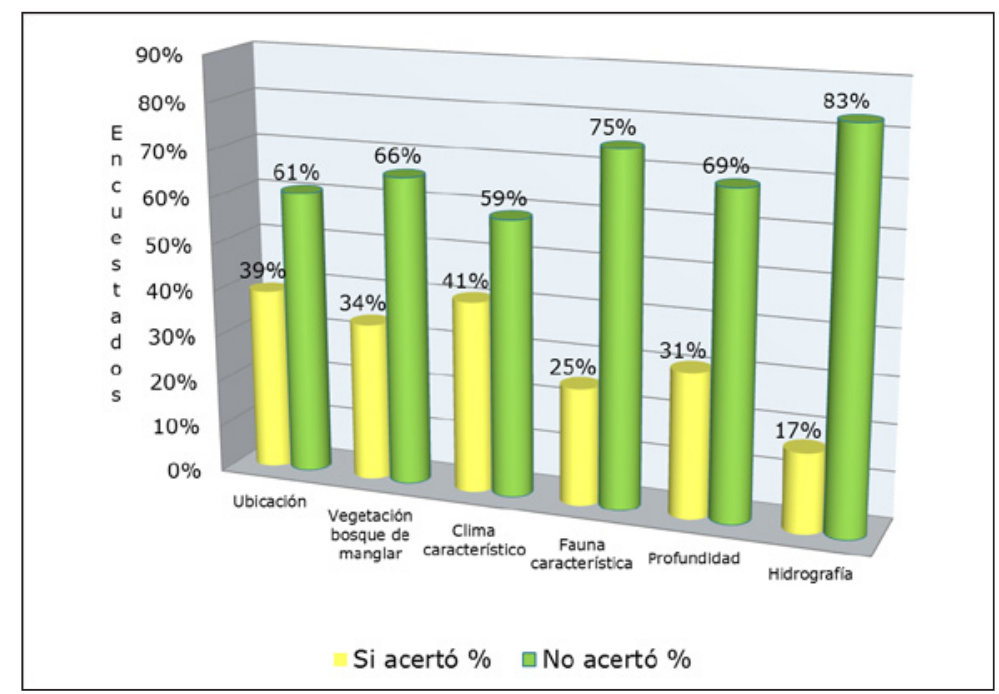

Figura 4. Dimensión: Nivel de conocimiento. Indicadores: Aspectos geográficos de la Laguna de las Peonías. Elaboración propia.

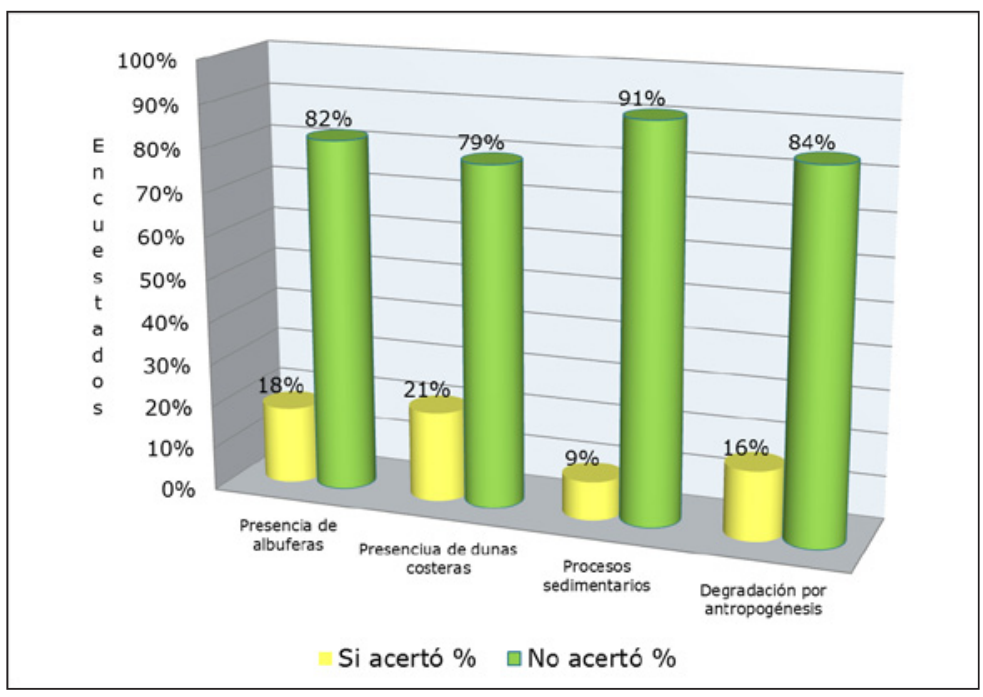

FiguRA 5. Dimensión: Nivel de conocimiento. Indicadores: Aspectos geomorfológicos de la Laguna de las Peonías. Elaboración propia. 
por parte de los estudiantes, según baremo de interpretación utilizado (tabla 3). Los resultados descritos, son una clara demostración de la ausencia de estrategias y recursos novedosos en la enseñanza de la Geografía Física local en los planteles de Educación Media General.

La tendencia reflejada en los resultados coloca de manifiesto por un lado, la exigua presencia de recursos didácticos basados en paisajes naturales para la enseñanza de procesos relacionados a la Geografía Física local, y, por el otro, la falta de motivación por parte de los educandos en asimilar conocimientos geográficos de sus espacios naturales cercanos. Las potencialidades que se exhiben en el escenario de la Laguna de las Peonías, representa en sí un paisaje con los ornamentos geográficos necesarios (características geográficas, aspectos geomorfológicos, degradación socio-ambiental, entre otros) para ser considerado un museo natural, el cual puede servir de insumo didáctico en la enseñanza de la Geografía.

La utilización de las referidas potencialidades en el proceso educativo, trae consigo ventajas de primer orden: motivan al educando en la exploración, observación y estudio de los elementos paisajísticos; al análisis sistémico del medio geográfico; y la integración holística de conocimientos sobre el espacio físico (Benayas, 1994; Labarca y Chourio, 2016). Por ende, las visitas guiadas cuentan con los elementos metodológicos y educativos necesarios que posibilitan al estudiante y al docente la capacidad de visualizar el ornamento natural de los paisajes visitados de manera concreta, mediante la implementación de rutas que sirven de guía en el estudio del escenario natural seleccionado, convirtiéndose éste en un gran museo natural para la enseñanza.

\subsection{Escenario de la Laguna de las Peonías como museo didáctico}

Entre los municipios Maracaibo y Mara del estado Zulia, al noroeste del estrecho de Maracaibo en la parroquia Idelfonso Vázquez (figura 1), se emplaza la Laguna de las Peonías, escenario natural de origen litoral situada astronómicamente entre las coordenadas $10^{\circ} 43^{\prime} 10^{\prime \prime}$ y $10^{\circ} 45^{\prime} 50^{\prime \prime}$ LN y $71^{\circ} 35^{\prime} 02$ » y $71^{\circ} 40>50$ » LW, contando con $5900 \mathrm{~m}$ de longitud, $2200 \mathrm{~m}$ de anchura y profundidad promedio de $0,70 \mathrm{~cm}$. Geomorfológicamente corresponde a la depresión del Lago de Maracaibo y es considerada uno de los reservorios naturales más importantes de la región zuliana, además que califica como uno de los humedales conteros continentales más prominentes de la geografía local (Mora, 2009).

El proceso geomorfológico que incide en la formación del escenario de la Laguna de las Peonías y le imprime su fisionomía particular, es la deriva litoral. En tiempo geológico pasado, en específico durante el Holoceno de la era Cenozoica y luego de las glaciaciones del Pleistoceno, se activaron los diversos procesos sedimentarios 
según los agentes exógenos. En lo que respecta a las zonas costeras, las olas son las responsables de arrastrar material sedimentario de las corrientes arriba a las corrientes bajas, desembocando en la acumulación de dicho material en las costas marinas, a este proceso de le denomina deriva litoral (Tarbuck y Lutgens, 2005). De allí se desprende, de entre tantas geoformas, la formación de albuferas o lagunas costeras, ejemplo de ello es la Laguna de las Peonías (figura 1).

En consideraciones de Mora (2009), Espinoza y Morales (2008) y Andrade (2007), el escenario del citado complejo lagunar posee potencialidades geográficas resaltantes como relieve plano de 0 a $20 \mathrm{~m}$ de altitud, con predominancia de orillas de playas e islotes de reciente formación; presencia de vegetación de bosque de manglares característica de los humedales costeros; clima de bosque seco tropical con variaciones térmicas de $24^{\circ}$ a $33^{\circ}$; hidrografía representada por cuatro (4) corrientes intermitentes denominadas caños o cañadas que bordean y desembocan en el espejo de agua (figura 1); procesos sedimentarias derivados de los caños que vierten en la laguna; litoral lacustre compuesto por suelos con textura franco arenoso; problemáticas ambientales por la intervención humana, entre otros.

Estas particularidades, convierten al escenario del geosistema lagunar de las Peonías en un verdadero museo natural propicio para la enseñanza de procesos y rasgos concernientes a la Geografía Física. Los ornamentos físico-naturales y socioambientales que tienen asidero en el referido espejo de agua, hacen de este espacio un escenario natural con múltiples facetas educativas, que ha de permitir la aprehensión de aprendizajes significativos a los estudiantes que lo visiten, siempre que cuenten con la adecuada estrategia aplicada por el docente guía.

Según los lineamientos de Benayas (1994), el escenario de las Laguna de las Peonías funge como museo natural didáctico puesto que es: motivar, ya que representa en sí un compendio de procesos y rasgos geográficos visibles que invitan al estudiante a observarlos, conocerlos, comprenderlos y entender su génesis; interdisciplinario, debido a que representa una porción espacial del geosistema local donde se pueden estudiar diversidad de conocimientos, entre los que destaca la geografía, geomorfología, climatología, ecología, urbanismo, entre otras; y encubridor de misterios, puesto que en su fisionomía pintoresca oculta un serie de información relacionada a aspectos geográficos, gemorfológicos y socio-ambientales que rigen su estructura y dinámica, lo cual se puede develar a los educandos mediante la implementación de una visita guiada al complejo lagunar.

\section{LA PROPUESTA}

Como parte de este estudio, se genera una propuesta de museo natural para la enseñanza de la Geografía Física, utilizando para ello las potencialidades geográficas 
del escenario de la Laguna de las Peonías. Para dinamizar la salida de campo al museo natural referido, se elabora una visita guiada como estrategia y recurso innovador para conectar al estudiante con el paisaje visitado. Las visitas guiadas, son un recurso efectivo en manos del docente ya que le permite planificar salidas de campo a lugares de interés académico y recreacional para el estudiantado, desde el cual van a obtener aprendizajes vivenciales y de manera diferente a los adquiridos en el aula de clases (Antúnez, 2003).

La misma autora, expone que al aplicar las visitas guiadas en las asignaturas relacionadas a las ciencias sociales y naturales, el docente puede alcanzar los objetivos de: a) despertar el interés en los estudiantes por aprendizajes vivenciales; b) propiciar la investigación científica en los estudiantes, c) estimular la participación de los educandos en la construcción de sus propias experiencias de aprendizaje al estar en contacto con la naturaleza, y d) contribuir en el mejoramiento de la enseñanza formal de las ciencias naturales y sociales, caso Geografía de Venezuela.

La elaboración de la visita guiada basada en las potencialidades del escenario de la Laguna de las Peonías como museo natural ubicado entre los municipios Maracaibo y Mara (figura 1), está compuesta por cinco (5) estaciones a cumplir en un día de salida de campo al complejo lagunar (figura 6), orientadas al estudio, comprensión y valoración de los rasgos geográficos, geomorfológicos y socio-ambientales presentes

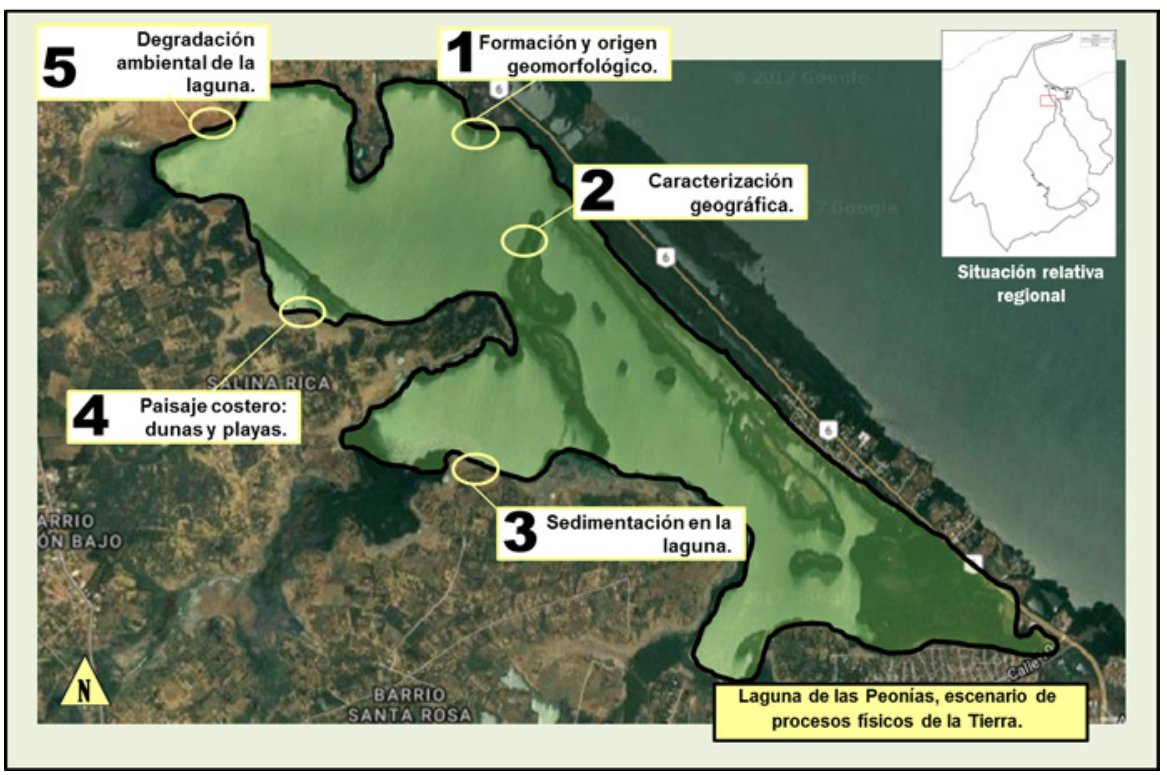

FIGURA 6. Estaciones definidas de la visita guiada al geosistema lagunar de Las Peonías.

Fuente: Imágenes de satélite de www.googlemaps.com; Inspección de campo de los autores (2017). Elaboración propia. 
en el área. La visita guiada, materializada de manera impresa como recurso didáctico, se intitula "Laguna de las Peonías, escenario de procesos físicos de la Tierra". Cada una de las estaciones está conformada por título, ubicación en el área de estudio, competencia de logro, materiales recomendados, aspectos teóricos a considerar y actividad de evaluación.

\subsection{Objetivos de la propuesta}

- Facilitar un recurso didáctico novedoso conformado por una visita guiada basada en el escenario de la Laguna de las Peonías que contribuya en la enseñanza y aprendizaje de características geográficas del medio físico en la asignatura Geografía de Venezuela.

- Aportar herramientas didácticas que incentiven en el estudiante la identificación de los rasgos geográficos exhibidos en el escenario de la Laguna de las Peonías.

- Fomentar en los estudiantes y docentes valores de sensibilización y preservación de los espacios naturales de Venezuela, caso Laguna de las Peonías.

\subsection{Descripción de la visita guiada sobre la Laguna de las Peonías}

Estación 1: Formación y origen geomorfológico (tabla 4).

\begin{tabular}{|c|c|c|}
\hline Ubic & petencia de logro & Materiales a utilizar \\
\hline & $\begin{array}{l}\text { el ori } \\
\text { cuya }\end{array}$ & $\begin{array}{l}\text { nes } \\
\text { guna, }\end{array}$ \\
\hline \multicolumn{3}{|c|}{ Aspectos a considerar } \\
\hline \multicolumn{3}{|c|}{$\begin{array}{l}\text {-Ubicación geográfica de la Laguna de las Peonías. } \\
\text {-El sistema lagunar Las Peonias se encuentra geográficamente ubicado en el sector noroeste del Lago de Maracaibo, al norte de la } \\
\text { parroquia Idelfonso Vázquez del municipio Maracaibo (figura 1) (Andrade, 2007). } \\
\text { La laguna cuenta con siete (7) islotes, de los cuales dos contienen tierra firma y los otros cinco son de manglares. Uno de los } \\
\text { islotes de tierra firme lleva por nombre "islote Zapato" (figura 1). } \\
\text {-Conceptualización de albuferas, origen e implicaciones geomorfológicas con la Laguna de las Peonías. } \\
\text {-Una albufera, denominada también laguna costera o lagoon según estimaciones de diversos autores, es una zona de agua poco } \\
\text { profunda que se sitúa en las costas sedimentadas, entre una isla de barrera y tierra firme (Strahler y Strahler, 1989). Po otro lado, } \\
\text { Tarbuck y Lutgens (2005), se refieren a las albuferas como lagunas de aguas tranquilas que separan la costa de una isla de } \\
\text { barrera, creada ésta por cretas de arena paralelas a la costa originadas por la sedimentación marina. } \\
\text {-La formación de un albufera, se genera cuando por la acción de la deriva litoral (depósito de los materiales arrastrados por las } \\
\text { corrientes marinas en las costas), los sedimentos que arrastran las olas se siguen acumulando en los alrededores de una flecha } \\
\text { marina (cordón de arena paralelo a la costa, unida a ella por uno de sus extremos), hasta el punto de que ésta se curva hacia el } \\
\text { litoral costero uniendo su otro extremo y resultando de alli la formación de un cordón o barra litoral, dejando asi encerrada un } \\
\text { porción de agua denominada albufera (Strahler y Strahler, 1989; Rivera, 2005). } \\
\text {-Un ejemplo claro de una albufera, lo constituye la Laguna de las Peonias, cuya fisionomia corresponde a una laguna costera de } \\
\text { origen litoral. Luego de las glaciaciones del Pleistoceno, según consideraciones de Tarbuck y Lutgens (2005), ocurrieron los } \\
\text { procesos sedimentarios que dieron origen a las formas de relieve deposicionales. Durante el Holoceno, las olas del Lago de } \\
\text { Maracaibo ejercieron su acción sedimentaria (deriva litoral) sobre las costas del suelo zuliano, formando así playas, flechas } \\
\text { litorales, barras litorales y albuferas a su alrededor. De este modo, quedaron configuradas las lagunas costeras del estado Zulia, tal } \\
\text { es el caso de la Laguna de las Peonias (figura 7). }\end{array}$} \\
\hline
\end{tabular}

TABLA 4. Estación 1 de la visita guiada. Elaboración propia. 
- Actividad de evaluación: Luego de la estación, se aplica a los estudiantes la técnica de la "Ilustración Descriptiva". Se hace entrega a cada participante de una copia del mapa satelital de la Laguna de las Peonías, y sobre él deben señalar: Ubicación geográfica, albufera y barra o cordón litoral, con respectivas definiciones según la explicación del docente guía y lo observado en el complejo lagunar.

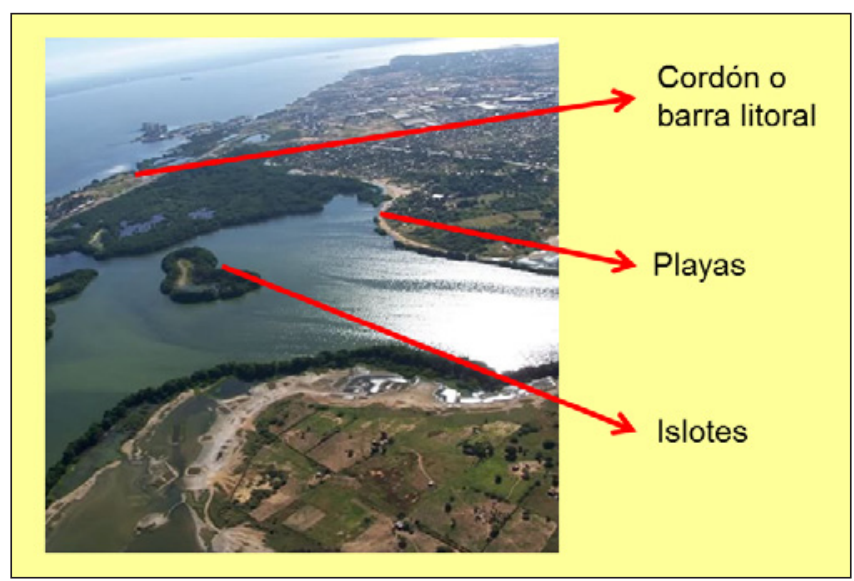

FiguRA 7. Morfología costera en la Laguna de las Peonías.

Elaboración propia.

\section{Estación 2: Caracterización geográfica (tabla 5).}

\begin{tabular}{|c|c|c|}
\hline Ubicación & Competencia de logro & Materiales a utilizar \\
\hline $\begin{array}{l}\text { Desde estación anterior, "Formación } \\
\text { y origen geomorfológico", andar en } \\
\text { lacha } 1 \mathrm{Km} \text { aproximadamente en } \\
\text { dirección sur-este hasta el islote } \\
\text { Zapato (figura 6). }\end{array}$ & $\begin{array}{l}\text { Al término de la estación, el educando estará } \\
\text { en capacidad de señalar los rasgos } \\
\text { geográficos que se exhiben en el escenario } \\
\text { Laguna de las Peonías. }\end{array}$ & $\begin{array}{l}\text { Libreta de anotaciones, regla, colores, mapa } \\
\text { de la laguna y cámara fotográfica. }\end{array}$ \\
\hline
\end{tabular}

Aspectos a considerar

-Identificación de las características físico-geográficas resaltantes que se exhiben en el escenario de la Laguna de las Peonías: relieve plano, vegetación de bosque hidrófilo (manglares), clima tropical, hidrografía intermitente y fauna característica.

-El geosistema lagunar de las Peonías, conforma un vaso hidráulico con una extensión aproximada de $5900 \mathrm{~m}$ de largo, $2200 \mathrm{~m}$ de ancho y profundidad promedio de $0,70 \mathrm{~cm}$. Presenta una fisionomía de relieve plano, con ausencia de pendientes y altitudes que no sobrepasan los 20m (Andrade, 2007, Mora, 2009), contando además con la presencia de islotes y playas de reciente formación

- La vegetación característica de la zona es de bosque hidrófilo, compuesto por una diversidad de tipos de manglares que adornan el sistema lagunar (figura 8). Según Mora (2009), los manglares presente en la referida laguna son el mangle blanco, mangle rojo, mangle negro y el de tipo botoncillo.

-El clima que se percibe en la Laguna de las Peonías es el perteneciente a la depresión del Lago de Maracaibo, de bosque seco tropical, con temperaturas oscilantes entre los $28^{\circ}$ y $33^{\circ}$.

-La hidrografía de la zona se considera intermitente, ya que cuenta con cuatro (4) cañadas o caños que vierten sus aguas en el espejo lagunar, en ausencia de cursos de agua naturales. Los caños se denominan Caño Irragory (nor-oeste), Caño Manfuey (oeste), Caño Fénix y Caño Araguato (sur) (figura 1); los tres primeros son aguas residuales pertenecientes a los centros poblados que la bordean, y el último, responsable del intercambio de agua con el Lago de Maracaibo. Según Espinoza y Morales (2008), la laguna se alimenta por la acción de las mareas del Lago de Maracaibo a través del Caño Araguato.

-Como fauna característica, en los espacios de la laguna perviven variedad de especias, tales como peces (corocoro, bocachico, lisa, bagre, entre otros); crustáceos (camarón blanco y cangrejo azul); aves (garcita blanca, cuervos de agua, patitos de agua zamuritos, gavilanes, yaguazas coloradas y flamingos rosados, siendo éstos últimos los más característicos); y reptiles (culebras de agua y tragavenados) (Andrade, 2007; Mora, 2009).

TABLA 5. Estación 2 de la visita guiada. Elaboración propia. 
- Actividad de evaluación: Terminada la explicación del docente, se solicita a los estudiantes que formen equipos de cuatro (4) personas. Utilizando la libreta de apuntes, cada equipo debe generar un "cuadro sinóptico de doble entrada", señalando relieve, vegetación, clima, hidrografía, fauna y profundidad de la Laguna de las Peonías. Archivar la actividad para su presentación final.

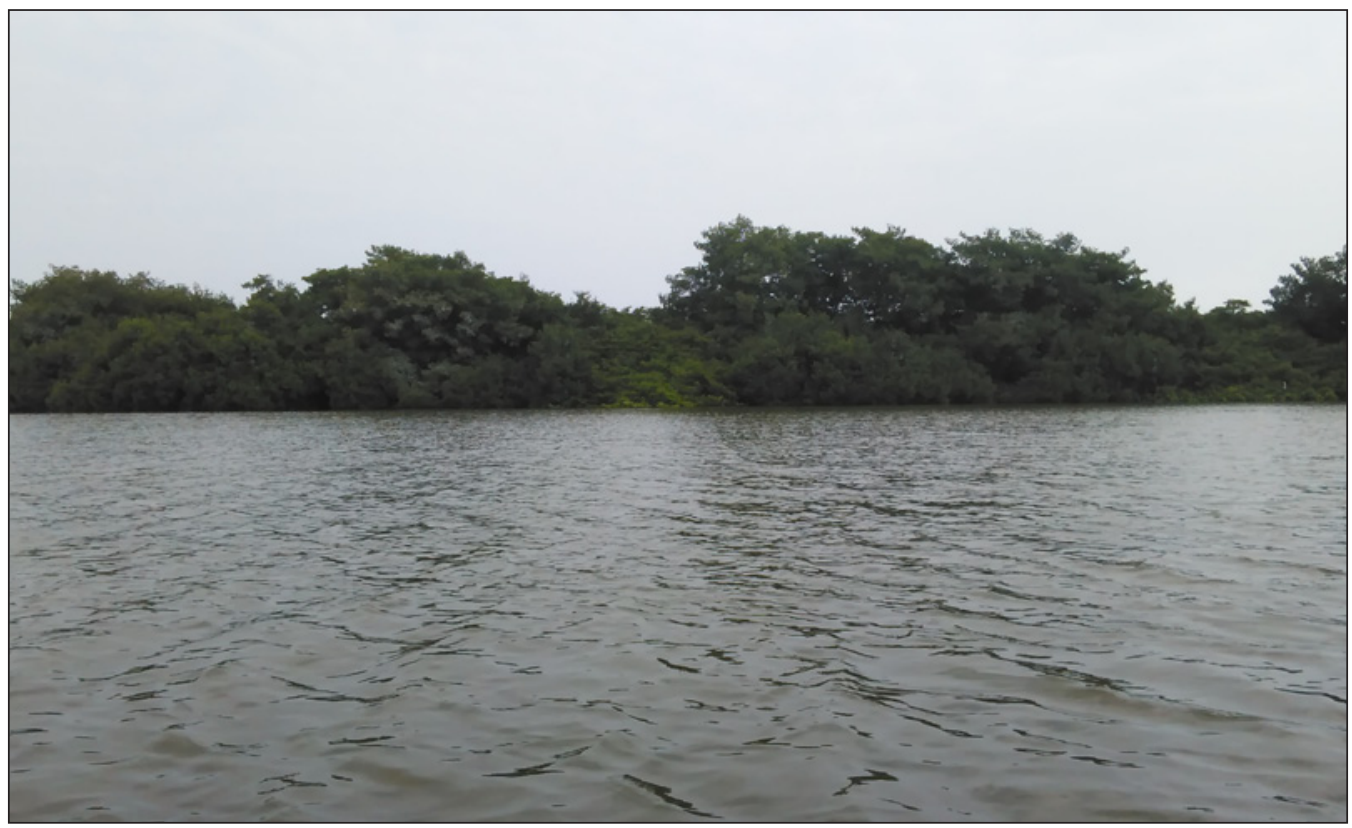

Figura 8. Presencia de manglares en Laguna de las Peonías. Tomada por los autores (2017).

Estación 3: Sedimentación en la laguna (tabla 6).

\begin{tabular}{|c|c|c|}
\hline Ubicación & Competencia de logro & Materiales a utilizar \\
\hline $\begin{array}{l}\text { Seguir recorrido en lancha desde la } \\
\text { estación "Caracterización } \\
\text { geográfica", en dirección sur-oeste, } \\
\text { hasta aproximadamente } 2,5 \mathrm{~km} \\
\text { llegando a tierra firme, al lado del } \\
\text { Caño Manfuey (figura 6). }\end{array}$ & $\begin{array}{l}\text { Culminada la estación, el estudiante logrará } \\
\text { explicar el proceso de sedimentación que } \\
\text { ocurre en el espejo lagunar de Las Peonías } \\
\text { como consecuencia del decantamiento de los } \\
\text { caños que lo bordean. }\end{array}$ & $\begin{array}{l}\text { Mapa de la laguna, libreta de anotaciones, } \\
\text { ilustraciones sobre sedimentación, cámara } \\
\text { fotográfica e hidratación. }\end{array}$ \\
\hline \multicolumn{3}{|c|}{ Aspectos a considerar } \\
\hline \multicolumn{3}{|c|}{$\begin{array}{l}\text {-Explicación del proceso de sedimentación que ocurre en los espacios de la Laguna de las Peonías a través de los caños o cañadas } \\
\text { que vierten sus aguas en ella. } \\
\text {-Andrade (2007), infiere que la laguna recibe grandes aportes de sedimentos a través de los caños o cañadas que la bordean } \\
\text { (Irragory, Manfuey y Fénix), generando de éste modo el proceso geodinámico de la sedimentación. } \\
\text {-La sedimentación, es el proceso geológico por medio del cual los materiales detríticos erosionados y transportados, son } \\
\text { depositados en un determinado lugar, que pueden ser fondos marinos, fondos lacustres, depresiones continentales, valles, entre } \\
\text { otros (Dávila, 2011). Para ilustrar, la Laguna de las Peonías funge como cuenca receptora de sedimentos, éstos provienen de los } \\
\text { materiales que arrastran los cursos intermitentes que la alimentan. }\end{array}$} \\
\hline
\end{tabular}

TABLA 6. Estación 3 de la visita guiada.

Elaboración propia. 
- Actividad de evaluación: Dadas las explicaciones de la estación, el docente solicita a los estudiantes la elaboración de la técnica del "Acróstico". A partir de la palabra Sedimentación, cada estudiante en su libreta de anotaciones debe explicar el proceso de acumulación de materiales en el fondo del espejo de agua a raíz del traslado de éstos por los caños o cañadas que bordean la laguna. Archivar la actividad para presentación final en el aula de clases.

Estación 4: Paisaje costero, dunas y playas (tabla 7).

\begin{tabular}{|l|l|l}
\hline \multicolumn{1}{c|}{ Ubicación } & \multicolumn{1}{c|}{ Competencia de logro } & Materiales a utilizar \\
\hline $\begin{array}{l}\text { A } 2 \mathrm{~km} \text { aproximadamente desde la } \\
\text { estación "Sedimentación en la } \\
\text { laguna", tomar dirección nor-oeste } \\
\text { hasta llegar a la playa del centro } \\
\text { poblado Salina Rica (figura 6). }\end{array}$ & $\begin{array}{l}\text { Explicada la estación, el educando sabrá } \\
\text { describir la génesis y morfología del paisaje } \\
\text { costero presente en la Laguna de las Peonías } \\
\text { compuesto por playas y dunas. }\end{array}$ & \begin{tabular}{l} 
bolsas de muestras y mapa de la laguna. \\
\hline \multicolumn{2}{c}{ Aspectos a considerar }
\end{tabular} \\
\hline
\end{tabular}

-Señalización y descripción de playas al oeste y norte de la Laguna de las Peonías (figura 6), morfológicamente de corta extensión y con litología compuesta por grava y arcilla limosa (figura 7). Una playa, es una acumulación de sedimentos que se encuentra a lo largo del borde litoral de un océano, lago o laguna (Tarbuck y Lutgens, 2005).

- Visualización de dunas costeras en el litoral oeste del geosistema lacustre de Las Peonías (figura 7), las cuales cuentan con un morfología alineada según la acción eólica, altitud de no más de dos metros y presencia de vegetación que irrumpe su movimiento. Gutiérrez (2008), afirma que las dunas litorales o costeras están constituidas por arenas procedentes de la playa y se cumulan por encima del nivel de marea alta, siendo influenciadas por la acción del viento que las moviliza y las nutre.

TABLA 7. Estación 4 de la visita guiada.

Elaboración propia.

- Actividad de evaluación: Al culminar la estación, el docente aplicará la técnica del "Registro fotográfico". Cada estudiante debe tomar fotografías al relieve costero observado, enfocándose en las dunas y playas. Las fotografías deben ser presentadas mediante un poster publicitario que posteriormente será divulgado vía redes sociales. Ésta actividad es para realizar en casa o en el aula, además debe ser archivada para la producción didáctica final.

Estación 5: Degradación ambiental de la laguna (tabla 8).

\begin{tabular}{|c|c|c|}
\hline Ubicación & Competencia de logro & Materiales a utilizar \\
\hline $\begin{array}{l}\text { A partir de la estación "Paisaje } \\
\text { costero, dunas y playas", recorrer } \\
\text { en lancha hacia el norte un } \\
\text { aproximado de } 2 \mathrm{~km} \text { hasta llegar al } \\
\text { caño Irragorry (figura 6). }\end{array}$ & $\begin{array}{l}\text { Una vez culminada la explicación de la } \\
\text { estación, el estudiante contará con la } \\
\text { capacidad de comprender los factores físicos } \\
\text { y humanos que inciden en la degradación } \\
\text { ambiental de la Laguna de las Peonías. }\end{array}$ & $\begin{array}{l}\text { Mapa de la laguna, libreta de anotaciones y } \\
\text { cámara fotográfica. }\end{array}$ \\
\hline
\end{tabular}

-Descarga de aguas negras, construcción de viviendas en zonas aledañas y acumulación de basura en los manglares, son los tres principales factores que inciden en el deterioro ambiental del espacio lacustre de Las Peonías, lo que denota en alto grado la intervención humana (antropogénesis).

-Bien es cierto que las descargas de agua por las cañadas que bordean la laguna son las responsables de que ocurra el proceso de sedimentación, pero también son las encargadas de desestabilizar las aguas de la laguna ocasionando cambios desfavorables en la calidad del agua que se almacena en ella.

-La construcción de viviendas en los alrededores de la laguna, le han proporcionado a éste una serie de problemáticas ambientales, entre las que destaca el incremento de desechos sólidos que son arrojados directamente en la laguna y la deforestación de la zona manglar.

-Por otro lado, la acumulación de basura en los manglares, causada por los asentamientos poblacionales, ha generado la deforestación de las áreas verdes que adornan el geosistema lagunar, la extinción de la fauna que vive en los mangles y la pérdida de espacio para su crecimiento.

TABLA 8. Estación 5 de la visita guiada.

Elaboración propia. 
- Actividad de evaluación: Luego de las disertaciones en la estación, el docente aplicará la técnica del "Mapa conceptual". En la libreta de apuntes, de manera individual los educandos deben elaborar un mapa conceptual sobre los tres principales factores que deterioran el equilibrio ambiental de la laguna. Todas las actividades hechas durante y después de la visita guiada, que corresponden a cada estación, se deben presentar mediante un Portafolio Didáctico, cuya ornamentación y estructura se discute en clases.

\section{CONCLUSIONES}

1. Al diagnosticar los recursos didácticos utilizados por los docentes en el proceso de enseñanza y aprendizaje de la asignatura Geografía de Venezuela en las entidades Liceo Privado "San José de Calasanz" y Unidad Educativa Colegio Adventista "Sierra Maestra", se constata que el 55\% de los estudiantes encuestados afirma que no son utilizados los "recursos preparados" y "recursos audiovisuales"; lo que denota un enfoque tradicional en la comunicación del saber. Resala además, que más del $80 \%$, coincide en indicar que el docente no hace uso de los "recursos dirigidos" y "recursos naturales", lo que coloca de manifiesto la ausencia de salidas de campo hacia paisajes naturales para la enseñanza de procesos geográficos.

2. En la búsqueda de precisar el nivel de conocimiento que poseen los estudiantes de la asignatura Geografía de Venezuela respecto a las potencialidades geográficas presentes en el escenario de la Laguna de las Peonías, se evidencia debilidad en el dominio del conocimiento según baremo de interpretación empleado, puesto que en los ítems que agrupan los indicadores "hidrografía", "fauna característica", "presencia de albuferas", "presencia de playas y dunas costeras" y "procesos de sedimentación", más del $75 \%$ de la población estudiantil encuestada afirma no conocer la geografía del referido paisaje zuliano. En base a ello, se estima la necesidad de utilizar el escenario de la Laguna de las Peonías como museo natural para la enseñanza de los procesos geográficos del medio, utilizando como recurso la visita guiada.

3. Se genera una propuesta basada en el escenario de la Laguna de las Peonías como museo natural y recurso didáctico en la enseñanza de la Geografía Física. La propuesta está conformada por una visita guiada, confeccionada de manera estructurada, estacionaria, creativa y adecuada a la población estudiantil a la cual está dirigida, contando con un total de cinco (5) estaciones a cumplir durante un día de salida de campo al complejo lagunar. Cada estación cuenta con su título, ubicación, competencia de logro, materiales a utilizar, aspectos teóricos a considerar y actividad de evaluación a cumplir durante y después de la visita. Se recomienda su aplicación en la enseñanza de la Geografía de Venezuela. 


\section{REFERENCIAS BIBLIOGRÁFICAS}

Andrade, L., 2007. Participación comunitaria en la solución de la problemática ambiental del sistema lagunar Las Peonías. Trabajo Especial de Grado para optar al título de Especialista Scientiarym en Educación Ambiental. Universidad Rafael Urdaneta. Maracaibo, Venezuela. Pp. 43-56.

Antúnez, J., 2003. Visitas guiadas como estrategia para gerenciar la actitud crítica y participativa en los educandos de la II Etapa de Educación Básica en al área de Ciencias de la Naturaleza y Tecnología. Trabajo Especial de Grado. Universidad Nacional Abierta. Barquisimeto, Venezuela. Pp. 15.

Barreto, B. y Bernal, J., 2016. "La península de Paraguaná: Un paisaje natural para la enseñanza de la morfología litoral en Ciencias de la Tierra”. Investigación y Formación Pedagógica, Revista del CIEGC, Vol. 2(4). Universidad Pedagógica Experimental Libertador - IMPM. San Cristóbal, Venezuela. Pp. 30-50.

Benayas,J., 1994. “Viviendoel paisaje”.Revista Biocenocis. Red Editorial Iberoamericana Andes, Vol. 18. Bogotá, Colombia. Pp. 1-18.

Briones, G., 1990. Métodos y Técnicas de Investigación para las Ciencias Sociales. 3era ed. Tomo I. México: Trillas. Pp. 106.

Chávez, N., 2007. Introducción a la Investigación Educativa. 4ta ed. Maracaibo, Venezuela: González, S.A. Pp. 52.

Crespo, J., 2012. "Un itinerario didáctico para la interpretación de los elementos físicos de los paisajes de las sierras de Guadarrama”. Didáctica Geográfica, No. 3. Universidad Complutense de Madrid, España. Pp. 15-34.

Dávila, J., 2011. Diccionario Geológico. Lima, Perú: Arth Grouting, S.A.C. Pp. 733.

Espinoza, N. y Morales, F., 2008. "Macroinvertebrados bentónitcos de la Laguna de las Peonías, estado Zulia, Venezuela”. Boletín del Centro de Investigaciones Biológicas, Vol. 42(3). Universidad del Zulia. Maracaibo, Venezuela. Pp. 345-363.

García, A.; Jiménez, J. y Rodríguez, E., 2009. "La enseñanza de la Geografía e Historia desde la localidad". Geoenseñanza, Vol. 14(1). Universidad de los Andes. Mérida, Venezuela. Pp. 109-150.

Gouveia, E.; Montiel, K. y Bejas, M., 2005. "Uso y abuso de los libros de texto en la enseñanza de la Geografía”. Geoenseñanza, Vol. 10(2). Universidad de los Andes, Venezuela. Pp. 173-186.

Gutiérrez, M., 2008. Geomorfología. Madrid, España: Pearson Educación, S.A. Pp. 415. Hernández, R.; Fernández, C. y Baptista, P., 2014. Metodología de la Investigación. 6ta ed. D.F., México: McGraw-Hill Interamericana Editores, S.A. Pp. 92, 217. 
Hurtado, J., 2010. Metodología de la Investigación. Guía para la Comprensión Holística de la Ciencia. 4ta ed. Caracas, Venezuela: Quirón. Pp. 395.

Incart, T.; Fuentelzas, C. y Pulpón, A., 2006. Elaboración y presentación de un proyecto de investigación y una tesina. España: Publicaciones de la Universidad de Barcelona. Pp. 69.

Labarca, R. y Chourio, M., 2016. "Laguna de Mucubají. Propuesta didáctica para la enseñanza de procesos geomorfológicos desde las Ciencias de la Tierra". Investigación y Formación Pedagógica, Revista del CIEGC, Vol.2(4). Universidad Pedagógica Experimental Libertador - IMPM. San Cristóbal, Venezuela. Pp. 6-29.

Montiel, K. y González, Y., 2012. "El paisaje natural de la Isla de Toas. Una propuesta didáctica”. Omnia, Año 18(2). Universidad del Zulia. Maracaibo, Venezuela. Pp. 47-66.

Mora, R., 2009. Variaciones en la composición, abundancia y biomasa del fitoplanton de la Laguna de las Peonías (estado Zulia, Venezuela). Trabajo de Ascenso para optar a la categoría de Profesor Titular. Facultad Experimental de Ciencias, Universidad del Zulia. Maracaibo, Venezuela. Pp. 17-22.

Negrete, A. y Montiel, K., 2016. "Isla de Toas. Una propuesta didáctica para la valoración del paisaje". REDHECS, Revista Electrónica de Humanidades, Educación y Comunicación Social, Nro. 22(11). Universidad Rafael Belloso Chacín. Maracaibo, Venezuela. Pp. 171-201.

Olave, D., 2005. "El Itinerario Didáctico: una propuesta metodológica para el análisis geo-histórico local”. Geoenseñanza, Vol. 10(2). Universidad de los Andes. Mérida, Venezuela. Pp. 197-208.

Pérez, H., 1981. El proceso de investigación. D.F., México: Limusa. Pp. 143.

Rivera, H., 2005. Geología General. 2da ed. Lima, Perú: Auspicio Académico, Universidad Nacional Mayor de San Marcos. Pp. 341.

Sabino, C., 2007. El proceso de investigación. Una introducción teórico práctica. Nueva edición actualizada. Caracas, Venezuela: PANAPO. Pp. 103.

Santiago, J.A., 2005. "La situación de la enseñanza de la Geografía en Venezuela, desde su práctica escolar cotidiana”. Geoenseñanza, Vol. 10(2). Universidad de los Andes. Mérida, Venezuela. Pp. 163.172.

Strahler, A. y Strahler, A., 1989. Geografía Física. Barcelona, España: Omega, S.A. Pp. $334,335,341$.

Tarbuck, E. y Lutgens, F., 2005. Ciencias de la Tierra. Una introducción a la geología física. 8va ed. Madrid, España: Pearson Educación, S.A. Pp. 569, 571, 672. 
Zoido, F., 2010. "El paisaje como patrimonio natural y cultural". Memorias del III Simposio sobre Estética del Entorno, Obra Pública y Paisaje. Centro de Estudios de Obras Públicas. Madrid, España. Pp. 255-270. 